\title{
Core Levels Algorithm for Optimization: Case of Microwave Models
}

\author{
Ali Haydar \\ Department of Computer Engineering \\ Girne American University \\ Mersin-10, Turkey \\ Ezgi Deniz Ülker \\ Department of Computer Engineering \\ European University of Lefke \\ Mersin-10, Turkey
}

\author{
Kamil Dimililer \\ Department of Electrical and Electronics Engineering \\ Girne American University \\ Mersin-10, Turkey \\ Sadık Ülker \\ Department of Electrical and Electronics Engineering \\ European University of Lefke \\ Mersin-10, Turkey
}

\begin{abstract}
Metaheuristic algorithms are investigated and used by many researchers in different areas. It is crucial to find optimal solutions for all problems under study especially for the ones which require sensitive optimization. Especially, for real case problems, solution quality and convergence speed of the algorithms are highly desired characteristics. In this paper, a new optimization algorithm called Core Levels Algorithm (COLA) based on the use of metaheuristics is proposed and analyzed. In the algorithm, two core levels are applied recursively to create new offsprings from the parent vectors which provides a desired balance on the exploration and exploitation characteristics. The algorithm's performance is first studied on some well-known benchmark functions and then compared with previously proposed efficient evolutionary algorithms. The experimental results showed that even at the early stages of optimization, obtained values are very close or exactly the same as the optimum values of the analyzed functions. Then, the performance of COLA is investigated on real case problems such as some selected microwave circuit designs. The results denoted that COLA produces stable results and provides high accuracy of optimization without high parameter dependency even for the real case problems.
\end{abstract}

Keywords-Metaheuristic algorithms; evolutionary algorithms; microwave circuits, optimization

\section{INTRODUCTION}

Solution of optimization problems is an interesting field of study for various areas such as array antenna synthesis [1]-[3], financial analysis [4], [5], error minimization and game programming [6], [7], microwave design [8]-[10] and data mining [11], [12]. Most of the related algorithms are motivated from the nature and are aimed to find near optimal solutions of given problems [13]-[17]. The performances of proposed algorithms are usually represented with their solution quality and convergence speeds. Each algorithm has several control parameters which are needed to be well tuned depending on the optimization problem in order to achieve better performance. This can be considered as a vital step in most of the cases and affects the exploration and exploitation characteristics of the algorithms [18]-[20]. The number of control parameters and their adjustment are quite deterministic for the performance of the algorithms. Typically, an algorithm that needs a few control parameters is assumed as a good choice in solving a given problem. However, in some cases, even the fine adjustment of control parameters is not sufficient to find the optimal points of the problems. In such problems, the exploration strategies of the algorithms may not be adequate to converge to the global optimum of the given problem. Therefore, some modifications of the algorithms are proposed to solve these specific problems [21]-[24]. Introduction of new optimizers is still an open area of research, because of the lack of an optimization algorithm that performs well in all fields. Some optimization algorithms perform well for some problems, while perform inadequate for other problems [25]. In the literature, many different optimization algorithms have been proposed to increase the solution quality for complex optimization problems with as little effort as possible.

In many microwave design problems, it is required to deal with some highly nonlinear objective functions with a large number of variables. In addition, gradient based algorithms cannot yield sufficient solutions in most of the cases since the optimization parameters in most of the problems are highly coupled with each other. Evolutionary algorithms are widely used when the analytical methods are insufficient to obtain appropriate solutions [26]-[28]. Although Genetic Algorithm (GA) is the first dominant evolutionary algorithm, which was applied on microwave and electromagnetic based problems, Differential Evolution (DE) and Particle Swarm Optimization (PSO) algorithms including their variants dominate the other evolutionary algorithms in this field [3], [29], [30].

In this paper, a new evolutionary algorithm called Core Levels Algorithm (COLA) is proposed to solve complex optimization problems including the design problems for microwave circuits. COLA uses the similar steps of evolutionary algorithms such as selection of candidates, generation of offsprings and replacement of the parents with the new offsprings which have better fitness values. In addition to that, COLA focuses on two core levels to obtain better offspring candidates by their parent vectors. A balance 
is achieved between the core levels to perform iterative exploitation in near optimal regions and to have better exploration in the search region. Moreover, the second core level of the algorithm is designed in such a way that it performs an exploration scheme which is centered on the selected vector and covers the whole domain of interest in order to solve different kind of optimization problems without modifying the algorithm.

Optimization characteristics of COLA were studied with the use of benchmark functions. In this study, the correlation between the number of selected parent vectors and the optimization capability was observed. Also, a comparative analysis of COLA with DE, PSO and Harmony Search (HS) algorithms was done for multimodal functions to observe the benefits of COLA. Another goal of the paper was to verify that COLA is applicable to the real case problems. Therefore, two microwave models were selected and optimized to achieve this goal.

The rest of the paper is organized as follows: Section II introduces the main concept of COLA in detail. Section III focuses on benchmark function results, real case microwave problems and discussions. Lastly, Section IV summarizes the obtained results and concludes the paper.

\section{CORE LEVELS AlgORITHM (COLA)}

The Core Levels Algorithm implements a new method to find the global optimum of a given function which is mainly based on the use of the balance between two core levels. This provides good exploration and exploitation characteristics of the algorithm. The pseudo code of COLA is shown in Fig. 1 and the detailed steps of COLA are explained in the following paragraphs:

- Initialize the population randomly using uniform distribution in the related domain of the optimization problem.

The algorithm has a few control parameters to be set which are $n p$; the number of elements in the population and $k$; the number of elements to be combined. COLA starts by initializing randomly the solution set of the optimization problem. The initialization can be defined as follows:

$x_{i, j}=x_{\min , j}+\operatorname{rand}[0,1]\left(x_{\max , j}-x_{\min , j}\right)$,

Where, $i \in\{1,2, \cdots, n p\}$ and $j \in\{1,2, \cdots, D\}$. Here, $D$ represents the dimension of given problem, $x_{\min , j}$ and $x_{\max , j}$ represent the lower and upper bounds for the $j$ th variable respectively and $x_{i, j}$ is the $j$ th component of the $i$ th solution vector.

- Evaluate the fitness of each element in the population.

- Select $k$ number of solutions according to their fitness values.

Using roulette wheel selection, $k$ number of solutions are selected randomly according to their fitness values.

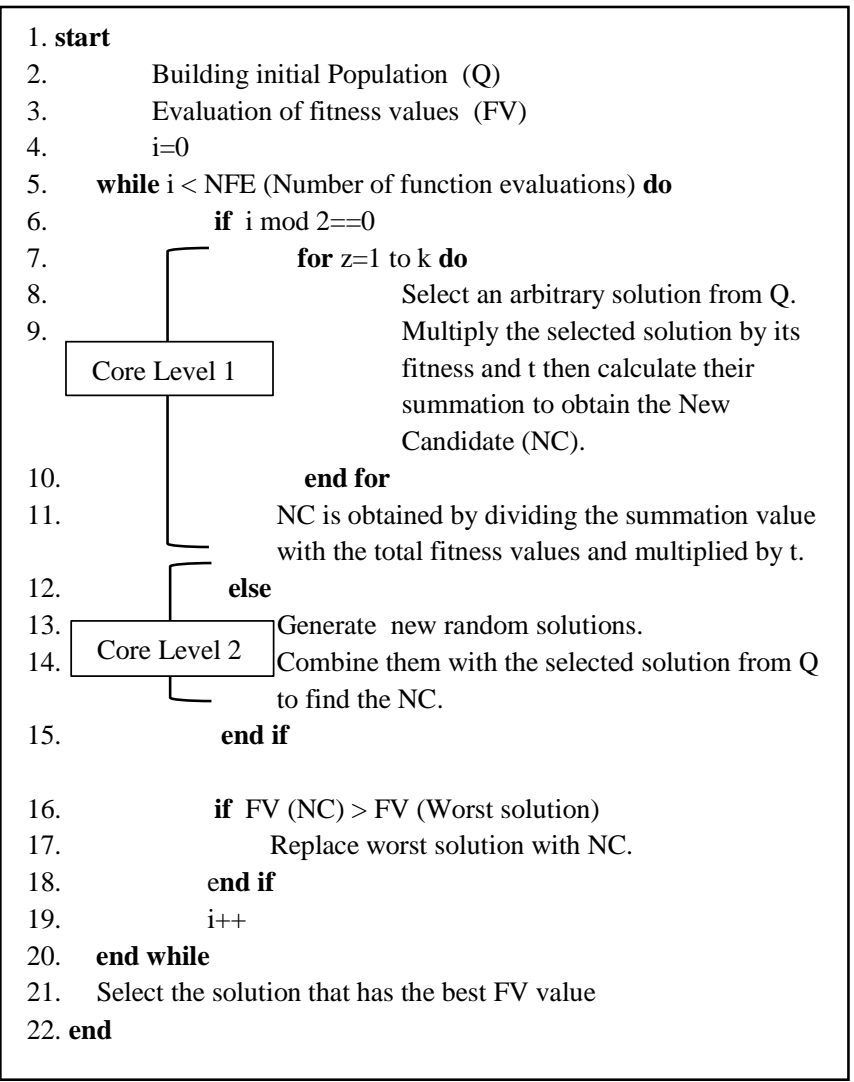

Fig. 1. Pseudo code of COLA.

- Obtain a new candidate solution from the selected solution and the randomly generated solution by using two core levels.

The new candidate solution vector is formed which is a weighted sum of the selected $k$ number of solution vectors by core level 1 . New candidate solution vector (NC) is calculated using the following expression:

$$
x_{j}^{\text {new }}=\frac{t_{1} \text { fit }_{1} x_{S_{1}, j}+t_{2} f i t_{2} x_{S_{2}, j}+\cdots+t_{k} f_{i} t_{k} x_{S_{k}, j}}{\sum_{n=1}^{k} t_{n} f i t_{n}},
$$

Where, $x_{j}^{\text {new }}$ is the $j$ th component of the new candidate vector, $t_{i} \in\{1,-1\}$ and is selected as -1 or 1 with equal probability, fit $_{p}$ is the fitness of the $p$ th selected solution vector and $x_{S_{p}, j}$ is the $j$ th component of the $p$ th selected vector. The new candidate formation using (2) is performed once in every two generations of the candidate vector.

Core level 2 is implemented for the next generation of the new candidate by using the following expression:

$$
x_{j}^{\text {new }}=(1-\alpha) x_{S, j}+\alpha x_{\text {rand }},
$$

where, $x_{j}^{\text {new }}$ is the $j$ th component of the new candidate vector, $\alpha$ is a random number in the uniformly distributed interval 
$[0,1], x_{S, j}$ is the $j$ th component of the randomly selected $S$ vector from the population and $x_{\text {rand }}$ is a randomly obtained number in the related domain which is calculated using (1). In the Core level 2, new vector $x_{j}^{\text {new }}$ can be obtained by applying in three different methods.

1) Only one of the randomly selected components of $x_{S}$ is updated.

2) The $k$ number of components in the range of $1<$ $k<D$ are updated where the number of $k$ is selected randomly.

3) All of the components of $x_{S}$ are updated by their linear combination with random numbers.

For each core level 2 application, only one of these methods is performed and this selection is done recursively.

- Replacement of the new candidate solution with the parent vector, if the fitness value of the new candidate is better.

The fitness of new vector is compared with the fitness of the worst parent solution vector in the population. If the fitness of new vector is better than fitness of the worst parent solution vector, then the new vector replaces with the worst parent solution vector in order to advance to the next generation. This can be expressed as follows:

$$
x^{\text {worst }}=\left\{\begin{array}{cc}
x^{\text {new }} & \text { if fitness }\left(x^{\text {new }}\right)>\text { fitness }\left(x^{\text {worst }}\right) \\
x^{\text {worst }} & \text { otherwise. }
\end{array}\right.
$$

- The steps will be repeated until the stopping criterion is met.

\section{EXPERIMENTAL RESULTS AND DISCUSSIONS}

\section{A. Testing with benchmark functions}

In this section, COLA is applied to 12 well-known benchmark functions to demonstrate its performance. These functions are taken from literature and they have been widely used for testing of optimization problems [31]. The selected benchmark functions are shown in Table 1. Among these functions, the first seven functions are unimodal functions and the following five functions are multimodal functions. For unimodal functions, convergence rates are the distinguishing characterictics of the optimization algorithms rather then final results. However, for multimodal functions, due to the many optimum points of problems, the final result obtained by algorithm is significant. The presented experimental results are average, standard deviation and the best value of the functions. All values are gathered over 40 independent runs. Average value indicates the solution quality, standard deviation value specifies the stability of the algorithm for undergoing random operations and the best value simply expresses the closest result to the optimal solution out of 40 independent runs.
TABLE I. The SELECTED Benchmark FunCtions UsEd IN EXPERIMENTS

\begin{tabular}{|c|c|c|c|}
\hline $\begin{array}{l}\text { Function } \\
\text { name }\end{array}$ & Function expression & Domain & $f_{\min }$ \\
\hline Sphere & $\sum_{i=1}^{n} x_{i}^{2}$ & {$[-100,100]$} & 0 \\
\hline $\begin{array}{l}\text { Schwefel } \\
2.22\end{array}$ & $\sum_{i=1}^{n}\left|x_{i}\right|+\prod_{i=1}^{n}\left|x_{i}\right|$ & {$[-10,10]$} & 0 \\
\hline $\begin{array}{l}\text { Schwefel } \\
1.2\end{array}$ & $\sum_{i=1}^{n}\left(\sum_{j=1}^{i} x_{j}\right)^{2}$ & {$[-100,100]$} & 0 \\
\hline $\begin{array}{l}\text { Schwefel } \\
2.21 \\
\end{array}$ & $\max \left\{\left|x_{i}\right|, 1 \leq i \leq n\right\}$ & {$[-100,100]$} & 0 \\
\hline Rosenbrock & $\sum_{i=1}^{n}\left[\begin{array}{l}100\left(x_{i+1}-x_{i}^{2}\right)^{2} \\
+\left(x_{i}-1\right)^{2}\end{array}\right]$ & {$[-30,30]$} & 0 \\
\hline Step & $\sum_{i=1}^{n}\left(\left\lfloor x_{i}+0.5\right\rfloor\right)^{2}$ & {$[-100,100]$} & 0 \\
\hline Quartic & $\sum_{i=1}^{n} i x_{i}^{4}+\operatorname{random}[0,1)$ & {$[-1.28,1.28]$} & 0 \\
\hline Schwefel & {$\left[\sum_{i=1}^{n}-x_{i} \sin \left(\sqrt{\left|x_{i}\right|}\right)\right]$} & {$[-500,500]$} & -12569.5 \\
\hline Rastrigin & $\sum_{i=1}^{n}\left[\begin{array}{l}x_{i}^{2}- \\
10 \cos \left(2 \pi x_{i}\right) \\
+10\end{array}\right]$ & {$[-5.12,5.12]$} & 0 \\
\hline Ackley & {$\left[\begin{array}{l}-20 \exp \left(-0.2 \sqrt{\frac{1}{n} \sum_{i=1}^{n} x_{i}^{2}}\right) \\
-\exp \left(\frac{1}{n} \sum_{i=1}^{n} \cos \left(2 \pi x_{i}\right)\right)+20+e\end{array}\right]$} & {$[-32,32]$} & 0 \\
\hline Griewank & {$\left[\begin{array}{l}\frac{1}{4000} \sum_{i=1}^{n} x_{i}^{2}- \\
\prod_{i=1}^{n} \cos \left(\frac{x_{i}}{\sqrt{i}}\right)+1\end{array}\right]$} & {$[-600,600]$} & 0 \\
\hline Penalized & 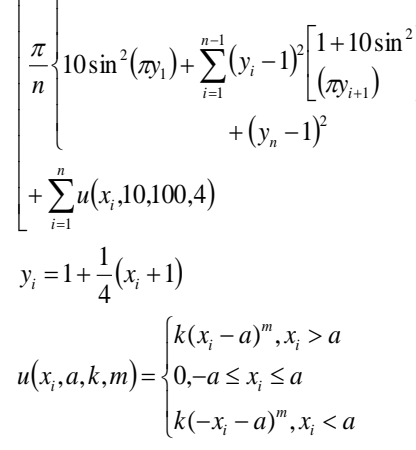 & {$[-50,50]$} & 0 \\
\hline
\end{tabular}

Population size is fixed to 100 , dimension is set to 30 and the number of function evaluations is set to 10000 for all benchmark functions. The algorithm continues until the stopping condition is met.

The results obtained for the listed functions above are given in Table 2. In this table, it is aimed to observe the performance of COLA for different $k$ parameter values in the set $\{3,4,5\}$ and a random selection of $k$ from the same set for each iteration. 
TABLE II. THE DESCRIPTIVE VALUES OF BENCHMARK FUNCTIONS BY SELECTED NuMBER OF $K$ PARAMETER AMONG THE PARENT VECTORS

\begin{tabular}{|c|c|c|c|c|c|}
\hline \multicolumn{2}{|c|}{$\begin{array}{c}10000 \\
\text { function evaluations }\end{array}$} & $k=3$ & $k=4$ & $k=5$ & $k=\operatorname{rand}[3-5]$ \\
\hline \multirow{3}{*}{ Sphere } & Avg & 0 & 0 & 0 & 0 \\
\hline & Best & 0 & 0 & 0 & 0 \\
\hline & Stdev & 0 & 0 & 0 & 0 \\
\hline \multirow{3}{*}{$\begin{array}{c}\text { Schwefel } \\
2.22\end{array}$} & Avg & 0 & 0 & 0 & 0 \\
\hline & Best & 0 & 0 & 0 & 0 \\
\hline & Stdev & 0 & 0 & 0 & 0 \\
\hline \multirow{3}{*}{$\begin{array}{c}\text { Schwefel } \\
1.2\end{array}$} & Avg & 0 & 0 & 0 & 0 \\
\hline & Best & 0 & 0 & 0 & 0 \\
\hline & Stdev & 0 & 0 & 0 & 0 \\
\hline \multirow{3}{*}{$\begin{array}{c}\text { Schwefel } \\
2.21\end{array}$} & Avg & 0 & 0 & 0 & 0 \\
\hline & Best & 0 & 0 & 0 & 0 \\
\hline & Stdev & 0 & 0 & 0 & 0 \\
\hline \multirow{3}{*}{ Rosenbrock } & Avg & 0.002908 & 0.000315 & 0.002061 & $1.92 \mathrm{E}-4$ \\
\hline & Best & $2.92 \mathrm{E}-27$ & $2.92 \mathrm{E}-27$ & $2.92 \mathrm{E}-27$ & $2.92 \mathrm{E}-27$ \\
\hline & Stdev & 0.007363 & 0.000647 & 0.000228 & 0.000227 \\
\hline \multirow{3}{*}{ Step } & Avg & 0 & 0 & 0 & 0 \\
\hline & Best & 0 & 0 & 0 & 0 \\
\hline & Stdev & 0 & 0 & 0 & 0 \\
\hline \multirow{3}{*}{ Quartic } & Avg & 0 & 0 & 0 & 0 \\
\hline & Best & 0 & 0 & 0 & 0 \\
\hline & Stdev & 0 & 0 & 0 & 0 \\
\hline \multirow{3}{*}{ Schwefel } & Avg & -12569.5 & -12569.5 & -12569.5 & -12569.5 \\
\hline & Best & -12569.5 & -12569.5 & -12569.5 & -12569.5 \\
\hline & Stdev & 0 & 0 & 0 & 0 \\
\hline \multirow{3}{*}{ Rastrigin } & Avg & 0 & 0 & 0 & 0 \\
\hline & Best & 0 & 0 & 0 & 0 \\
\hline & Stdev & 0 & 0 & 0 & 0 \\
\hline \multirow{3}{*}{ Ackley } & Avg & $-1.4 \mathrm{E}-16$ & $-1.4 \mathrm{E}-16$ & $-1.4 \mathrm{E}-16$ & $-1.4 \mathrm{E}-16$ \\
\hline & Best & $-1.4 \mathrm{E}-16$ & $-1.4 \mathrm{E}-16$ & $-1.4 \mathrm{E}-16$ & $-1.4 \mathrm{E}-16$ \\
\hline & Stdev & 0 & 0 & 0 & 0 \\
\hline \multirow{3}{*}{ Griewank } & Avg & 0 & 0 & 0 & 0 \\
\hline & Best & 0 & 0 & 0 & 0 \\
\hline & Stdev & 0 & 0 & 0 & 0 \\
\hline \multirow{3}{*}{ Penalized } & Avg & $3.67 \mathrm{E}-6$ & $2.58 \mathrm{E}-6$ & $2.18 \mathrm{E}-6$ & $1.74 \mathrm{E}-6$ \\
\hline & Best & $1.36 \mathrm{E}-22$ & $1.36 \mathrm{E}-22$ & $1.36 \mathrm{E}-22$ & $1.36 \mathrm{E}-22$ \\
\hline & Stdev & $3.2 \mathrm{E}-06$ & $1.38 \mathrm{E}-6$ & $1.09 \mathrm{E}-6$ & $8.43 \mathrm{E}-7$ \\
\hline
\end{tabular}

It can be observed from the results in Table 2, except Rosenbrock, Ackley and Penalized functions, all other functions are converged to their optimal values regardless of selection of $k$. Moreover, when the results of Rosenbrock and Penalized functions are investigated, instead of selecting $k$ parameter as a fixed number, the selection of it randomly in given interval for each iteration decreases the average and standard deviation values. This is the indication of better solution quality and faster convergence speed of the algorithm under the random selection of $k$ parameter in the given interval. For these two functions, the average and standard deviation are shown for different values of $k$ in Fig. 2 and 3, respectively.

The figures illustrate that selecting parameter $k$ randomly in a given interval for each iteration presents better results. It is obvious that COLA performs effectively to reach to the optimum points of Rosenbrock and Schwefel functions. Since the control parameter $k$ is randomized in the given interval, COLA performs efficiently by using only a single control parameter which is $n p$; the number of elements in the population.

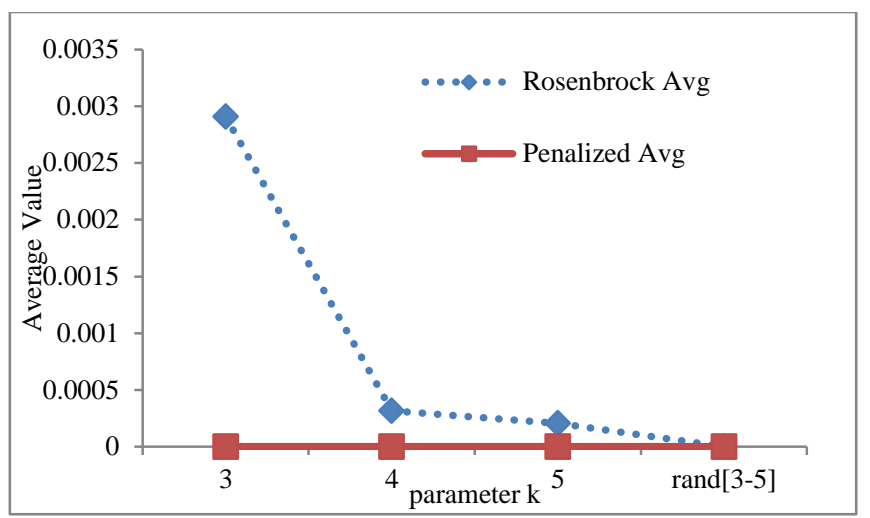

Fig. 2. Average function values obtained for Rosenbrock and Penalized functions for different values of $k$ parameter.

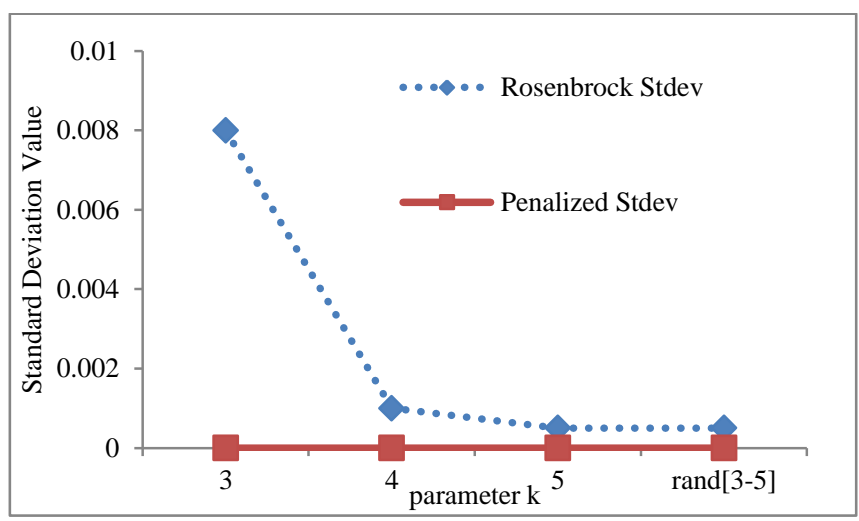

Fig. 3. Standard deviation of function values obtained for Rosenbrock and Penalized functions for different values of $k$ parameter.

In order to verify the advantage of COLA over some effective evolutionary algorithms which are Differential Evolution, Particle Swarm Optimization and Harmony Search algorithms, it is compared for the multimodal benchmark functions under the same conditions. These comparative results are presented in Table 3. The results for DE, PSO and HS algorithms are taken from the previously reported work [32]. Since the global optimum point is located in many local optimum points, it is quite challenging to reach the global optimum of given multimodal functions. It is also known that the solution quality of the last value achieved by an algorithm is a distinguishing characteristic especially for multimodal functions. Therefore, a comparison analysis is performed only for the multimodal functions. It can be seen from Table 3 that COLA optimizes the multimodal functions with a good convergence rate, while the others are away from the global points of the functions even after 10000 number of function evaluations.

\section{B. Microwave Models}

Importance of optimization and computer design has been realized for many years. One of the earliest papers on the area of optimization methods for microwave circuits was Bandler and Macdonald's work [33], [34]. A classical paper on the analysis part of microwave circuits in computer aided design was introduced by Monaco and Tiberio [35]. 
TABLE III. COMPARATIVE VALUES OF ALGORITHMS FOR MULTIMODAL FUNCTIONS.

\begin{tabular}{|l|l|l|l|l|l|}
\hline \multicolumn{2}{|l}{ Function name } & COLA & DE & PSO & HS \\
\hline \multirow{4}{*}{ Schwefel } & Avg & -12569.5 & -7485.74 & -8531.08 & -12554.6 \\
\cline { 2 - 6 } & Best & -12569.5 & -8128.58 & -10353.9 & -12566.1 \\
\cline { 2 - 6 } & Stdev & 0 & 270.62 & 949.247 & 28.8299 \\
\hline \multirow{4}{*}{ Rastrigin } & Avg & 0 & 137.899 & 46.3655 & 18.1256 \\
\cline { 2 - 6 } & Best & 0 & 126.013 & 19.0816 & 12.7443 \\
\cline { 2 - 6 } & Stdev & 0 & 6.6812 & 17.416 & 3.41769 \\
\hline \multirow{5}{*}{ Griewank } & Avg & $-1.4 \mathrm{E}-16$ & 16.7758 & 2.86698 & 1.09805 \\
\cline { 2 - 6 } & Best & $-1.4 \mathrm{E}-16$ & 15.1746 & 0.65150 & 0.56317 \\
\cline { 2 - 6 } & Stdev & 0 & 0.75719 & 1.01934 & 0.29879 \\
\cline { 2 - 6 } & Avg & 0 & 1.53190 & 0.39352 & 1.04977 \\
\cline { 2 - 6 } & Best & 0 & 1.29684 & 0.05316 & 0.56317 \\
\hline \multirow{5}{*}{ Penalized } & Stdev & 0 & 0.19544 & 0.31861 & 0.0222 \\
\cline { 2 - 6 } & Avg & $1.74 \mathrm{E}-6$ & 5.08107 & 4.36306 & 0.29210 \\
\cline { 2 - 6 } & Best & $1.36 \mathrm{E}-22$ & 2.79334 & 0.37862 & 0.04269 \\
\cline { 2 - 6 } & Stdev & $8.43 \mathrm{E}-7$ & 2.13136 & 2.94708 & 0.24432 \\
\hline
\end{tabular}

In this paper, different methods used in the analysis programs of linear circuits in frequency domain were described. Also, determination of sensitivity and convenience of using one or the other method in relation to the number of parameters and different analysis methods were explained.

Different methodology which used the combination of experimental design and computer-aided design was demonstrated in [36]. In 2002, computer-aided design summary of works to date is included as a survey paper [37]. It is also indicated that there are three essential reasons for simulation of radio frequency and microwave circuits; to understand the physics of a complex system of interacting elements, to test new concepts and to optimize the designs. Over the years many papers for the computer aided design or optimization of microwave circuits can be found. Recently a new technique for rapid multi-objective optimization of the compact microwave passive components was presented [38], [39].

\section{Microwave Tapered Matching Circuit Design}

Microwave matching networks are important in the design of many different types of microwave circuitry. Only with proper matching such a circuit can attain maximum power transfer and eliminate the reflection. In microwave matching circuit design, especially when one needs to match real load impedances, one of the most useful network is a tapered microwave matching network which can be considered as a series of cascaded quarter wavelength transmission lines. The design for tapered lines is usually done by using computer algorithms for continuous sections [40], [41]. For this kind of structure design optimization using nature-inspired metaheuristic methods, namely, particle swarm optimization, was done [10]. An example circuit is shown in Fig. 4.

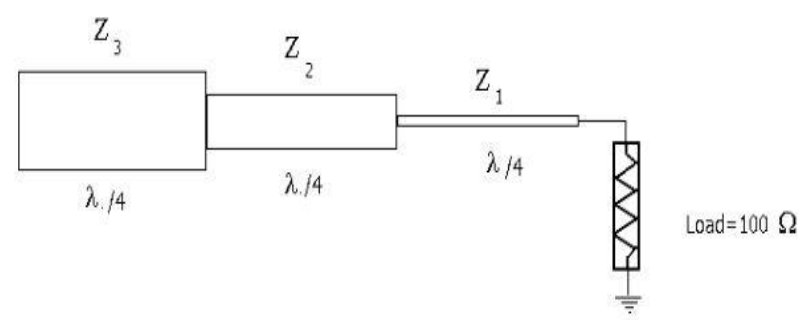

Fig. 4. Microwave Tapered matching network.

As can be seen from Fig. 4, the tapered line in this model of study consists of a series of $\lambda / 4$ transmission lines. This type of configuration can be used to achieve a match for real loads. In the model, $100 \Omega$ load is matched to a $50 \Omega$ line using a series of cascaded three transmission lines of a quarter wavelength long. Starting from the load higher impedance transmission lines exist and as moving along the tapered line, lower impedance transmission lines are obtained. The objective function of this circuit is given as below:

$$
f\left(Z_{1}, Z_{2}, Z_{3}\right)=\frac{1}{100}\left[\frac{Z_{1} \cdot Z_{3}}{Z_{2}}\right]^{2}-50,
$$

Where, the values of parameters, which are the characteristic impedances, $Z_{1}, Z_{2}$ and $Z_{3}$ must to be found also with the condition that $Z_{3}<Z_{2}<Z_{1}$ and the values are restricted to be in the range [0-100]. In order to observe the performance of COLA on this microwave circuit, 40 independent runs were performed. For each run, 1000 function evaluations were executed to obtain values of $Z_{1}, Z_{2}$ and $Z_{3}$ that provide optimal value of function (5). From the performed 40 independent trials, a sample of five impedence values $(\Omega)$ are shown in Table 4.

The reflection coefficient obtained by using first and the second trial values are plotted in Fig. 5 for the design frequency of $5 \mathrm{GHz}$ to demonstrate the impedance values. As it can be seen in Fig. 5, reflection coefficient values are around $-35 \mathrm{~dB}$ and below at $5 \mathrm{GHz}$, which indicates a good matching. This indicates that the values obtained by COLA are all correct yielding proper designs at the end.

TABLE IV. IMPEDENCE VALUES FOR FIVE INDEPENDENT TRIALS

\begin{tabular}{|l|l|l|l|}
\hline & $\boldsymbol{Z}_{\mathbf{1}}(\boldsymbol{\Omega})$ & $\boldsymbol{Z}_{\mathbf{2}}(\boldsymbol{\Omega})$ & $\boldsymbol{Z}_{\mathbf{3}}(\boldsymbol{\Omega})$ \\
\hline Trial 1 & 81.809594 & 50.364347 & 43.531533 \\
\hline Trial 2 & 93.715554 & 63.237186 & 47.714004 \\
\hline Trial 3 & 82.929957 & 61.988993 & 52.855251 \\
\hline Trial 4 & 82.588533 & 66.591554 & 57.014379 \\
\hline Trial 5 & 92.639060 & 57.197776 & 43.658620 \\
\hline
\end{tabular}




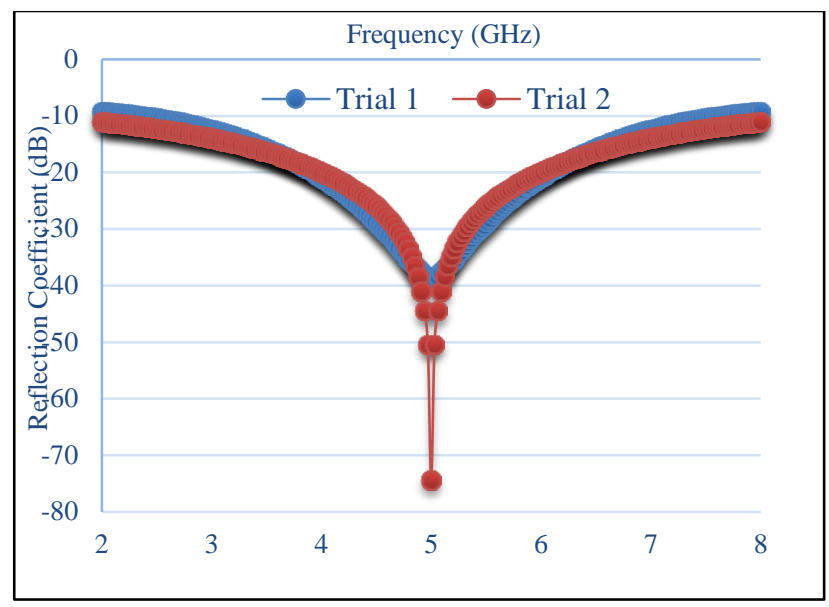

Fig. 5. Reflection coefficient values for two different trial sets.

Another aspect that needs to be analyzed is the sensitivity analysis of the obtained results using the algorithm. Simple $5 \%$ error is introduced to the obtained values to see how the design is affected. In this case it is observed that there is not a change in design operating frequency since the lengths are not different. The reflection value is worse, however still lower than $-25 \mathrm{~dB}$ which yields a reasonable match since the reflection coefficient value is very low.

\section{Microwave Amplifier Design}

Amplification is necessary for most of the electronic circuits and for microwave circuit systems. Nowadays, with the development of transistor technology, all microwave amplifiers use transistor devices which are more reliable and rugged. The main advantage of using transistor devices is that they can easily be integrated into monolithic circuits. Design of amplifiers in general requires the matching network design for input and output parts of the network. If the work is done by hand, first the stability of the transistor is checked and drawing the Gain Circles and selecting optimum points, one can perform the design operation using Smith Chart. This process can be performed by using metaheuristic algorithms especially the nature-inspired metaheuristic algorithms. Similar works, using metaheuristic algorithms to solve amplifier design problems, were performed by the following researchers for the given specific problems [9], [42]-[46]. Simple two-port microwave network which produces amplification with a proper design is shown in Fig. 6 .

In the Fig. 6, there are two matching nework designs that should be done simultaneously to achieve the desired gain. The overall design also needs a compromise in gain and in return loss at the same time. The design in this case is the design of two impedance matching networks to achieve the desired gain goal. In other words, the design requires finding the proper lengths of transmission lines $\mathrm{d}_{1}, \mathrm{~d}_{2}, \mathrm{l}_{1}$ and $\mathrm{l}_{2}$ at the central operation frequency. The power function that needs to be optimized is given by the following expression:

$$
G_{T}=\frac{\left|S_{21}\right|^{2}\left(1-\left|\Gamma_{S}\right|^{2}\right)\left(1-\left|\Gamma_{L}\right|^{2}\right)}{\left|\left(1-S_{11} \Gamma_{S}\right)\left(1-S_{22} \Gamma_{L}\right)-\left(S_{12} S_{21} \Gamma_{S} \Gamma_{L}\right)\right|^{2}}-16 .
$$

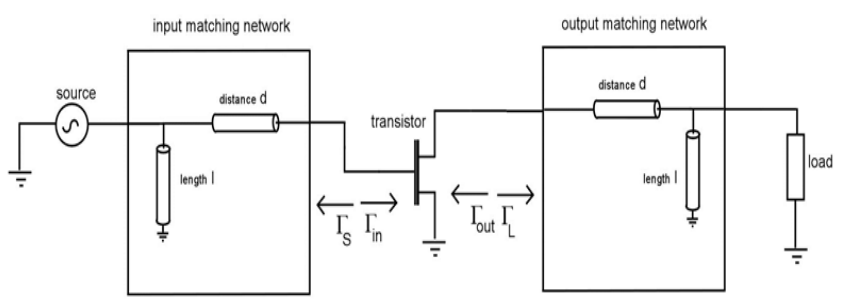

Fig. 6. Microwave amplifier design illustration.

This expression depends on transistor s-parameters: $S_{11}$, $S_{12}, S_{21}$ and $S_{22}$. The reflection parameters are $\Gamma_{S}$ and $\Gamma_{L}$ for source and load respectively. Expressions for $\Gamma_{\mathrm{S}}$ and $\Gamma_{\mathrm{L}}$ and $Z_{1}, Z_{2}, Z_{3}$ and $Z_{4}$ were derived in [9] and are as follows:

$$
\begin{aligned}
& \Gamma_{S}=\frac{Z_{2}-1}{Z_{2}+1}, \\
& \Gamma_{L}=\frac{Z_{4}-1}{Z_{4}+1}, \\
& Z_{2}=\frac{\operatorname{Re}\left(Z_{1}\right)+j\left(Z o \tan \left(d_{1}\right)+\operatorname{Im}\left(Z_{1}\right)\right)}{Z o-\operatorname{Im}\left(Z_{1}\right) \tan \left(d_{1}\right)+j \operatorname{Re}\left(Z_{1}\right) \tan \left(d_{1}\right)}, \\
& Z_{1}=\frac{j Z o \tan \left(l_{1}\right)}{1+j \tan \left(l_{1}\right)}, \\
& Z_{4}=\frac{\operatorname{Re}\left(Z_{3}\right)+j\left(Z o \tan \left(d_{2}\right)+\operatorname{Im}\left(Z_{3}\right)\right)}{Z o-\operatorname{Im}\left(Z_{3}\right) \tan \left(d_{2}\right)+j \operatorname{Re}\left(Z_{3}\right) \tan \left(d_{2}\right)}, \\
& Z_{3}=\frac{j Z o \tan \left(l_{2}\right)}{1+j \tan \left(l_{2}\right)},
\end{aligned}
$$

Where, $Z o$ is the characteristic impedance of the transmission line. In our design, transistor FHX35X, manufactured by Fujitsu Cooperation was used. With the chosen s-parameters, the design was centered at frequency of $10 \mathrm{GHz}$. The design was optimized to get a gain of 16 , which in decibels is $12 \mathrm{~dB}$. The characteristics were gathered over 40 independent runs. Each run had 1000 function evaluations. The values for $d_{1}, d_{2}, l_{1}$ and $l_{2}$ were restricted to be range [0, $2 \pi]$. These values for $d_{1}, d_{2}, l_{1}$ and $l_{2}$ were obtained in terms of radians and five of them were tabulated in Table 5. In addition, a microwave simulator was used to obtain characteristics. Fig. 7 demonstrates results of Trial 1 for gain and reflection values.

The plot shows $S_{11}$ and $S_{22}$ which are the reflections in the ports 1 and 2, respectively and $S_{21}$ which is the transmission from port 1 to 2 . In this case, gain value is $S_{21}$ value. In a design, especially at the design frequency $S_{11}$ and $S_{22}$ values should be minimized and if possible $S_{11}$ and $S_{22}$ values should be kept below $0 \mathrm{~dB}$ at all times to avoid oscillations. It is seen that this is roughly happening all throughout the band of observation from $7 \mathrm{GHz}$ to $13 \mathrm{GHz}$. 
TABLE V. A SAMPLE SOLUTION SET FOR DIFFERENT NUMBER OF TRIALS

\begin{tabular}{|l|l|l|l|l|}
\hline & $\mathbf{d}_{\mathbf{1}}$ & $\mathbf{d}_{\mathbf{2}}$ & $\mathbf{l}_{\mathbf{1}}$ & $\mathbf{l}_{\mathbf{2}}$ \\
\hline Trial 1 & 4.279915 & 4.826180 & 5.600057 & 5.307381 \\
\hline Trial 2 & 1.077591 & 3.406553 & 2.602834 & 4.395524 \\
\hline Trial 3 & 0.905713 & 1.858479 & 2.642391 & 2.087756 \\
\hline Trial 4 & 0.069484 & 4.511318 & 0.641231 & 2.218899 \\
\hline Trial 5 & 3.332904 & 3.381557 & 3.622599 & 1.301090 \\
\hline
\end{tabular}

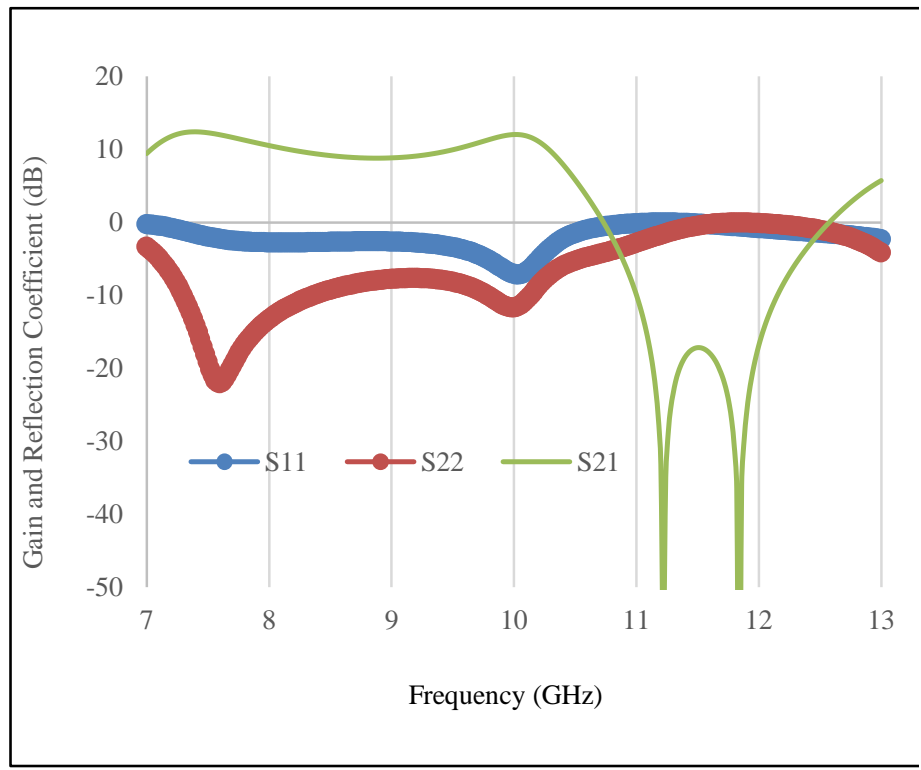

Fig. 7. Gain and reflection coefficient values of Trial 1 for the amplifier design.

At the design frequency $S_{11}$ value is $-8 \mathrm{~dB}$ and $S_{22}$ value is $-11 \mathrm{~dB}$. The gain value is also at $12 \mathrm{~dB}$. A sensitivity test is also performed to see if the values produced by the algorithm are sensitive to errors. $3 \%$ error is introduced to all of the length values, the overall gain dropped to $10.77 \mathrm{~dB}$; however the simulation indicated that the transistor is stable. When a $5 \%$ error is introduced to all of the length values, the maximum gain dropped from $12 \mathrm{~dB}$ to $9.82 \mathrm{~dB}$ which is not a very desirable feature. However this kind of result is expected, since the gain performance in the model relies on correct transmission line lengths and introducing a 5\% error in all lengths is actually a significant change in the design. These results overall indicate that COLA performed efficiently and it can be a good candidate of optimization algorithm for designing a stable amplifer.

\section{CONCLUSIONS}

In this paper, a new optimization algorithm COLA is proposed to find the global optimum points of given problems by providing good solution quality with robust solutions to the random operations in the algorithm. Two core levels are applied simultaneously to reach these goals by providing a balance between exploration and exploitation characteristics.

The algorithm is tested with different characteristics of benchmark functions, compared with powerful evolutionary algorithms and then applied to two real microwave circuit design problems. The results obtained for benchmark functions indicate that COLA provides better solution quality than the analyzed algorithms and its convergence speed is fairly good even for the first stages of optimization such as 10000 function evaluations. The results for microwave circuits obtained by COLA are verified by microwave simulators and it is seen that it produces accurate results. It is studied that the control parameter $k$ is randomized in given interval produces better results. Therefore, COLA uses only one control parameter $n p$ which is not necessary to be well tuned for the problems studied in this paper. The number of population $n p$ is fixed to 100 for all the problems in this study. In other words, it can be considered that the algorithm is almost parameter free which can be used for any problem without tuning any control parameter. This advantage of the algorithm makes it very practical to be used for any real case problems.

According to these results, it can be concluded that the solution quality of the algorithm is better than the analyzed algorithms and also is quite robust even though there is no parameter to be tuned. The algorithm COLA can be suggested as a candidate for optimization problems including real case problems from different fields. As future work, a new research can be done to compare COLA with recent hybrid optimization algorithms.

\section{REFERENCES}

[1] Y. Chen, S. Yang and Z. Nie, The Application of a modified differential evolution strategy to some array pattern synthesis problems, IEEE Trans. Antennas and Propagation, 56, pp. 1919-1927, 2008.

[2] B. Choudhury, S. Manickam and R.M. Jha, Particle Swarm Optimization for Multiband Metamaterial Fractal Antenna, J. Optim. 2013, pp. 1-8, 2013.

[3] E.D. Ulker, A. Haydar and K. Dimililer, Application of Hybrid Optimization Algorithm in the Synthesis of Linear Antenna Array, Math. Prob. Eng. 2014, pp. 1-7, 2014.

[4] P. Wen-Tsao, A new Fruitfly Optimization Algorithm: Taking a Financial Distress model as an Example, Knowledge-Based Systems, 26, pp. 69-74, 2012.

[5] E. Hadavandi, H. Shavandi, A. Ghanbari, Integration of Genetic Fuzzy Systems and Artificial Neural Networks for Stock Price Forecasting, Knowledge-based Systems. 23, pp. 800-808, 2010.

[6] R. Köker, Genetic Algorithm Approach to a Neural-Network based inverse kinematics solution of robotic manipulators based on error minimization, Spec. Sect. on New Trends in Ambient Intelligence and Bio-inspired Syst. 222, pp. 528-543, 2013.

[7] Z.W. Geem, Harmony Search Algorithm for Solving Sudoku, Knowledge-based Intelligent Information and Engineering Systems, Lect. Notes Comput. Sci. 4692, pp. 371-378, 2007.

[8] E.D. Ulker and S. Ulker, Application of Particle Swarm Optimization to Microwave Tapered Microstrip Lines, Int. J. Comput. Sci. Eng (CSEIJ) 4, pp.59-64, 2014.

[9] E. Deniz, S.Ulker, Clonal selection algorithm application to simple microwave matching network, Microw. Opt. Technol. Lett. 53, pp. 991993, 2011.

[10] S. Ulker, Broadband Microwave Amplifier Design Using Particle Swarm Optimization, J. Comput. 6, pp. 2272-2276, 2011.

[11] A.A. Freitas, Data mining and knowledge discovery with evolutionary algorithms. Springer Sciene and Business Media, 2013.

[12] A. Mukhopadhyay, U. Maulik, S. Bandyopadhyay, C.A.C Coello, A survery of multiobjective evolutionary algorithms for data mining: Part I, IEEE Trans. on Evol. Comput.. 18, pp. 4-19, 2014.

[13] Z.W. Geem, J.H. Kim and G.V. Loganathan, Harmony Search Optimization: Application to Pipe Network Design, J. Model. Optim. 22, pp. 60-68, 2002. 
[14] J. Kennedy, R. Eberhart, Particle Swarm Optimization, IEEE International Conference on Neural Networks. pp.1942-1948, 1995.

[15] R. Storn, K. Price, Differential Evolution: A Simple and Efficient Heuristic for Global Optimization over Continuous Spaces, J. Glob. Optim. 11, pp.341-359, 1997.

[16] L.N. De Castro, F.J. Von Zuben, Learning and optimization using the clonal selection principle, IEEE Trans. Evol. Comput. 6, pp. 239-251, 2002.

[17] J.H. Holland, Adaptation in Natural and Artificial Systems, University of Michigan Press, Ann Arbor, Michigan, 1975.

[18] Y. Shi and R. Eberhart, Comparing Inertia Weights and Constriction Factors in Particle Swarm Optimization, Proc. Congr. Evol. Comput., pp. 84-88, 2000.

[19] A.S.D. Dymond, A.P. Engelbrecht and P.S. Heyns, The sensitivity of single objective optimization algorithm control parameter values under different computational constraint, IEEE Congress Evol. Comput. pp. 1412-1419, 2011.

[20] S.K. Smit and A. Eiben, Comparing Parameter Tuning Methods for Evolutionary Algorithms, IEEE Congress on Evol. Comput., pp. 399406, 2009.

[21] M. Mahdavi, M. Fesanghary and E. Damangir, An Improved Harmony Search Algorithm for Solving Optimization Problems, Appl. Math. Comput. pp. 1567-1579, 2007.

[22] Ghosh, S. Das, A. Chowdhury and R. Giri, An Improved Differential Evolution Algorithm with Fitness-based Adaptation of the Control Parameters, Infor. Sci., pp. 3749-3765, 2011.

[23] M.M. Ali, P. Kaelo, Improved particle swarm optimization for global optimization, App. Math. Comput. 196, pp. 578-593, 2008.

[24] Lihu, S. Holban, A Study on the Minimal Number of Particles for a Simplified Particle Swarm Optimization Algorithm, 6th IEEE International Conference on Applied Computational Intelligence and Informatics. pp. 299-303, 2011.

[25] D.H. Wolpert and W.G. Macready, No Free Lunch Theorem for Optimization, IEEE Trans. Evol. Comput. 1, pp. 67-82, 1997.

[26] Hoorfar, Evolutionary Programming in Electromagnetic Optimization: A Review, IEEE Trans. Antennas and Propagation. 55, pp. 523-537, 2007.

[27] L. Zhang L, P.H. Aaen, T. Dhaene, A. Sahu and V. Devabhaktuni, An Introduction to Evolutionary Optimization for Microwave Engineering, Wiley Encyclopedia of Electrical and Electronics Engineering, 1999.

[28] S.K. Goudos, K. Siakavara, T. Samaras, E.E. Vafiadis and J.N. Sahalos, Self Adaptive Differential Evolution Applied to Real-valued Antenna and Microwave Design Problems, IEEE Trans. Antennas and Propagation, 59, pp.1286-1298, 2011.

[29] C. Lin, A. Qing and Q. Feng, Synthesis of Unequally Spaced Antenna Arrays by using Differential Evolution, IEEE Trans. Antennas and Propagation. 58, pp.2553-2561, 2010.

[30] S.K. Goudos, C. Kalialakis and R. Mittra R, Evolutionary Algorithms Applied to Antennas and Propagation: A Review of State of the Art, International J. Antennas and Propagation, 1, pp.1-12, 2016.
[31] X. Yao, Y. Liu and G. Lin G, Evolutionary Programming Made Faster, IEEE Trans. Evol. Comput. 3, pp. 82-102, 1999.

[32] E.D. Ulker and A. Haydar, A Hybrid Algorithm based on Differential Evolution, Particle Swarm Optimization and Harmony Search Algorithms, IEEE International Conference on Computer Science and Information Systems, pp. 417-420, 2013.

[33] J.W. Bandler, Optimization Methods for Computer Aided-Design, IEEE Trans. Microw. Theory Tech. 17, pp. 533-552, 1969.

[34] J.W. Bandler, P.A. MacDonald, Optimization of microwave networks by razor search, IEEE Trans. Microw. Theory Tech. 17, pp.552-562, 1969.

[35] V.A. Monaco and P. Tiberio, Computer-Aided Analysis of Microwave Circuits, IEEE Trans. Microw. Theory Tech. 22, pp. 249-263, 1974.

[36] J. Carrol, K. Chang, Statistical computer-aided design for microwave circuits, IEEE Trans. Microw. Theory Tech. 44, pp.24-32, 1996.

[37] M. Steer, Computer-Aided Design of RF and Microwave Circuits and Systems, IEEE Trans. Microw. Theory Tech. 50, pp. 996-1005, 2002.

[38] S. Koziel, A. Bekasiewicz, Rapid Simulation-Driven Multiobjective Design Optimization of Decomposable Compact Microwave Passives, IEEE Trans. Microw. Theory Tech. 64, pp. 2454-2461, 2016.

[39] S. Koziel, A. Bekasiewicz, Rapid Microwave Design Optimization in Frequency Domain using Adaptive Response Scaling, IEEE Trans. Microw. Theory Tech. 64, pp. 2749-2757, 2016.

[40] C. Marcu, A.M. Niknejad, A $60 \mathrm{GHz}$ High-Q Tapered Transmission Line Resonator in $90 \mathrm{~nm}$ CMOS, IEEE Microwave International Symposium Digest, pp. 775-778, 2006.

[41] D.I.L De Villiers, P.W. Van Der Walt and P. Meyer, Design of conical transmission line power combiners using tapered line matching sections, IEEE Trans. Microw. Theory Tech. 56, pp. 1478-1484, 2008.

[42] Sallem, B. Benhala, M. Kotti, M. Fakhfakh, A. Ahaitofuf A and M. Loulou, Application of swarm intelligence techniques to the design of analog circuits: evaluation and comparison, Analog Integrated Circuits Signal Process. 75, pp. 499-516, 2008.

[43] S. Ulker, Design of Low Noise Amplifiers Using Particle Swarm Optimization, Int. J. Artif. Intell. Appl. 3, pp. 99-106, 2012.

[44] O.J. Ushie, M. Abbod, E.C. Ashigwuike and S. Lawan, Constrained Nonlinear Optimization of Unity Gain Operational Amplifier Filters Using PSO, GA and Nelder-Mead, Int. J. Intell. Control Syst. 20, pp. 26-34, 2015.

[45] F. Gunes, S. Demirel, P. Mahouti, A simple and efficient honey bee mating optimization approach to performance characterization of a microwave transistor for the maximum power delivery and required noise, Internat. J. Numer. Model. Electron. Networks, Devices, Fields, 29, pp. 4-20, 2015.

[46] F. Gunes, S. Demirel, S. Nesil, Design Optimization of LNAs and Reflect array Antennas Using the Full-Wave Simulation-Based Artificial Intelligence Models with the Novel Metaheuristic Algorithms, Simulation-Driven Model Optim. 153, pp. 261-298, 2016. 